From the Laboratory of Psychopharmacology, University of Maryland, College Park, Maryland

\title{
Morphine Self-Administration, Food-reinforced, and Avoidance Behaviors in Rhesus Monkeys*
}

\author{
By \\ Travis Thonpson ** and Chartes R. Schuster
}

With 4 Figures in the Text

(Received March 11, 1963)

Research techniques based upon the behavioral principles of operant conditioning have provided a complementary approach to the standard. pharmacologic analysis of physical dependence upon opiates (SEEvers 1936, 1958). With these techniques, a simple arbitrary response is conditioned by following it with a reinforcement. For example, a food deprived rhesus monkey learns to press a lever because pressing a lever leads to a pellet of food. By the same token, if a lever pressing response is followed by the injection of an opiate to a physically dependent animal, that response will be learned.

Using the general principle outlined above, several investigators have recently demonstrated that it is possible to condition physically dependent rats to emit operant responses for morphine reinforcement. Nichols and co-workers $(1956,1959)$, have shown that dependent rats will learn to drink water containing morphine rather than the initially preferred morphine-free tap water. WEEKs $(1960,1962)$ has recently shown that unrestrained rats can be conditioned to emit a lever-pressing response to receive intravenously infused morphine on a fixed ratio reinforcement schedule. In addition, he has shown that pretreatment with nalorphine produces an increase in rate of responding for the drug, presumably produced by the induced abstinence syndrome.

\section{General procedure}

The experimental approach we have used is an extension of the lines of investigation based upon the principle of reinforcement of behavior by opiate administration, to permit an analysis of the interaction of pharmacological and behavioral variables in opiate dependence (ScHUSTER

* This research was supported by research grant MY-1604 from the National Institute of Mental Health and grant NsG 189-61 from the National Aeronautics and Space Administration, to the University of Maryland.

** National Science Foundation post-doctoral fellow 41088.

Psychopharmacologia, Bd. 5 
and Thompson, 1962). Rhesus monkeys seated in restraining chairs obtain all of their food and avoid painful electric shocks by pressing levers under specific visual or auditory stimulus conditions. When a yellow light is present, pressing the lever leads to food. When a clicker is presented, pressing the lever postpones an electric shock. The animals are in the experiment for 24 hours a day. so that large and continuous samples of the animal's behavior can be brought under experimental control. Interspersed with periods when the animal is working for food or avoiding shock, occasions are provided when a response will lead to the infusion of morphine sulphate through a chronically indwelling jugular catheter. Since the various stimuli associated with food, shock and drug periods, as well as the drug administration and data recording are all controlled electronically by devices located in an adjacent control room, it is not necessary to disturb the animal for the measurement of drug-effects or to administer the opiate. Because of this degree of experimental control, it is possible to obtain highly reliable measures of behavioral output from day to day over a period of 6 months. Thus, the experimenter has at his disposal a double bladed instrument for the analysis of drug-behavior interaction. It is possible to measure the effects of conditions of the opiate administration on food and shockavoidance behaviors, and at the same time to measure the animal's disposition to administer the drug to himself.

Experiment1. In this preliminary study, the feasibility of conditioning monkeys to work for a morphine reinforcement was established. These experiments employed 3 adult male rhesus monkeys weighing approximately $3.5 \mathrm{~kg}$. Following surgical implantation of the jugular catheter (Niemann, Schuster and Thompson 1962), the animals were infused 4 times daily with $7 \mathrm{mg}$ of morphine sulphate in isotonic saline, for a period of 30 days. Food and water were available at all times. Following this period during which physical dependence was developed, the monkeys were conditioned to emit a specific behavioral sequence in order to obtain subsequent intravenous infusions of morphine. The final series of behaviors required is called a fixed interval-fixed ratio chain (FI-FR chain). The fixed interval component is indicated by the onset of a tone. The first oceurrence of a response after two minutes in the presence of the tone turned on a white light signaling a change to a fixed ratio schedule. A fixed ratio of 25 responses in the presence of the white light produced the infusion of $7 \mathrm{mg}$ of morphine over a period. of 60 seconds. During the infusion of morphine a red light was presented. Thus, in order to obtain the drug, the animal must first complete the two-minute fixed interval giving it the opportunity to emit 25 more responses to be followed by morphine infusion. Each 24 hour experimental session was divided into 4 cycles of 6 hours duration. In each 
cycle the subjects had the opportunity to self-administer $1 / 4$ of its total daily morphine intake of $28 \mathrm{mgs}$ or $8.0 \mathrm{mg} / \mathrm{kg}$.

Following stabilization of the FI-FR behavior sequence, the infusion and stimulus presentation apparatus was turned off for 24 hours. The

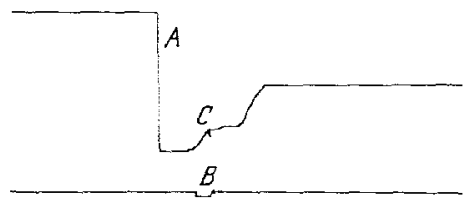

Last FI-FR prior to nalorphine pretreatment

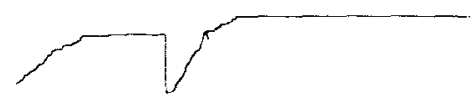

$1.0 \mathrm{mg}$ nalorphine 45 minutes pretreatment

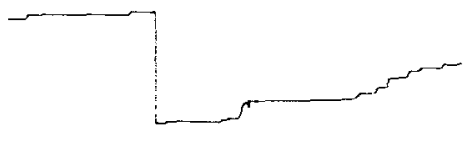

6 hours 45 minutes following nalorphine pretreatment

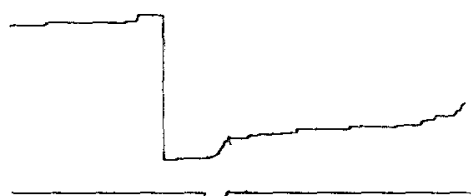

12 hours 45 minutes following nalorphine pretreatment

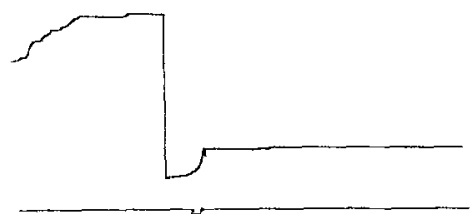

Last FI-FR prior to morphine deprivation

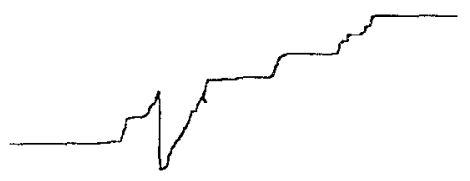

First FI-FR following 24 hours morphine deprivation

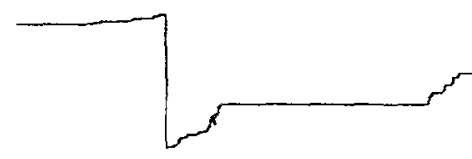

Second FI-FR following 24 hours morphine deprivation

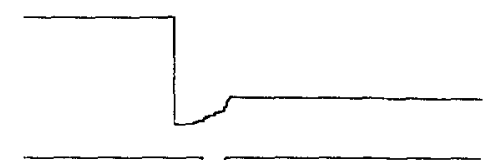

Third FI-FR following 24 hours morphine deprivation

$A$ Reset of response pen indicating the onset of 2 mimute FI with accompanying tone. $B$ Deflection of event marker indicating the FR, period with acempanying white light. $Q$ Brief deflection of response pen indicates infusion of morphine and presentation of a red light

Fig. 1. Representative cumulative response records of fixed interval-fixed ratio performance following 24 hours of morphine deprivation compared FI-FR performance 45 minutes after the administration of $1.0 \mathrm{mg}$ nalorphine, i.v.

following day the 6 hour FI-FR schedule was reinstated and changes in behavior as a function of the increased deprivation condition were observed. Fig. 1 presents cumulative response records for the session following 24 hours deprivation of morphine. The number of responses in the fixed interval are dramatically increased as compared with the baseline condition. It can also be seen that the latency to completion of the 25 responses in the FR is reduced. After the FI-FR behavior 
had reached the pre-deprivation baseline, $1.0 \mathrm{mg}$ of nalorphine was administered I.V. 45 minutes prior to the scheduled FI-FR period. As can be seen in Fig. 1, the number of responses in the fixed interval is markedly increased. In addition, the time taken to completion of the ratio is much shorter than any of the baseline periods. Subsequent stabilization of the FI.FR chain was followed by a series of sessions on which the animals were pretreated with $7.0,14.0$ or $21.0 \mathrm{mg}$ of morphine 45 minutes before a FI-FR period. The effect of morphine pretreatment is illustrated in Fig. 2. As can be seen, there is a progressive disruption in the tendency to work for the drug as an increasing function of dose. These effects have been replicated on three different animals.

Experiment 2. Subsequent experiments have interspersed food and shock-avoidance periods between the four drug-reinforced behavior periods. Each six hour cycle consisted of 4 shock avoidance periods, 4 food periods and 1 drug reinforced FI-FR period.

The presentation sequence of these periods was as follows: Food, Shock, Food, Shock, FI-FR drug period, Shock, Food, Shock, Food. A minimum of 8 and maximum of 32 minutes separated the presentation of the various periods.

The food period consisted of five successive fixed ratios of thirtyfive responses. If the animal failed to complete the five ratios the food period was automatically terminated after 8 minutes.

The shock avoidance periods were presented for a maximum of 8 minutes or until the animal received 5 shocks. The shock schedule consisted of a 10 second warning clicker presented on a variable time schedule with an average interval of 60 seconds. Responses between the warning stimulus presentations had no consequence. Responses in the warning stimulus terminated the clicker and avoided the 0.5 second electric shock. The $1.5 \mathrm{ma}$ electric shock was delivered to the subject through eight stainless steel electrodes mounted on the inside of a leather waist belt.

Fig. 3 shows baseline performance over a period of 30 days, for food reinforced responses, shock avoidance responses, responses in the fixed interval leading to the opportunity to work for morphine, and the lenght of time to complete a ratio of 25 responses to be infused with morphine. The effects of abstinence from the drug (by not providing the opportunity to work for the drug) on food-reinforced and shock-avoidance behaviors were superimposed upon this stable baseline performance. Fig. 4 presents the changes in the food reinforced and shock-avoidance behaviors during 48 hours of abstinence. As can be seen, there is a progressive reduction in tendency to work for food, and the shock-avoidance latencies increase. Following the first drug infusion after 4.8 hours of abstinence, the food reinforced behavior returns immediately, and the shock- 


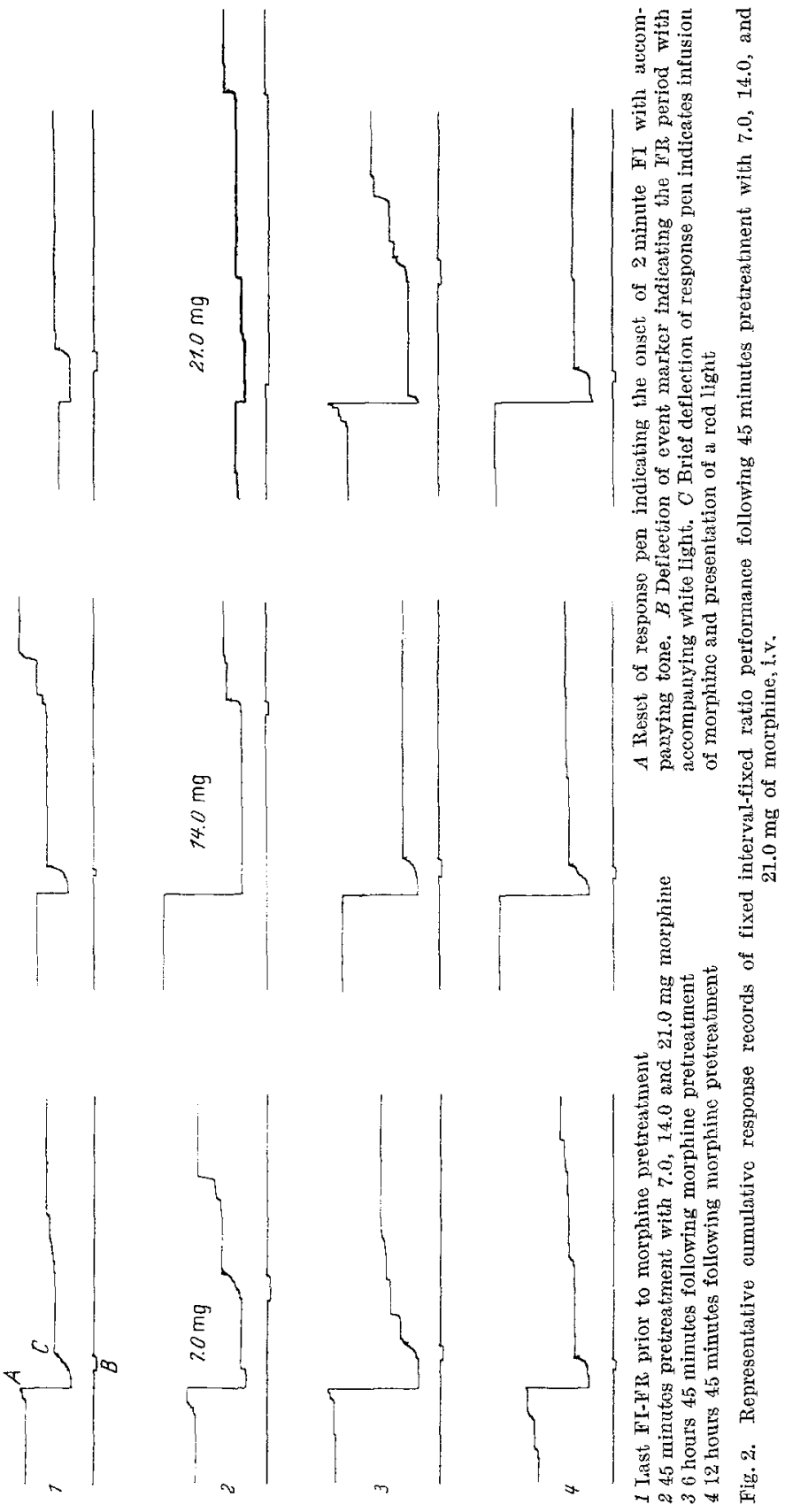

avoidance latencies begin decreasing toward the pre-abstinence baseline. This effect has been replicated on three animals over a period of 4 months, 


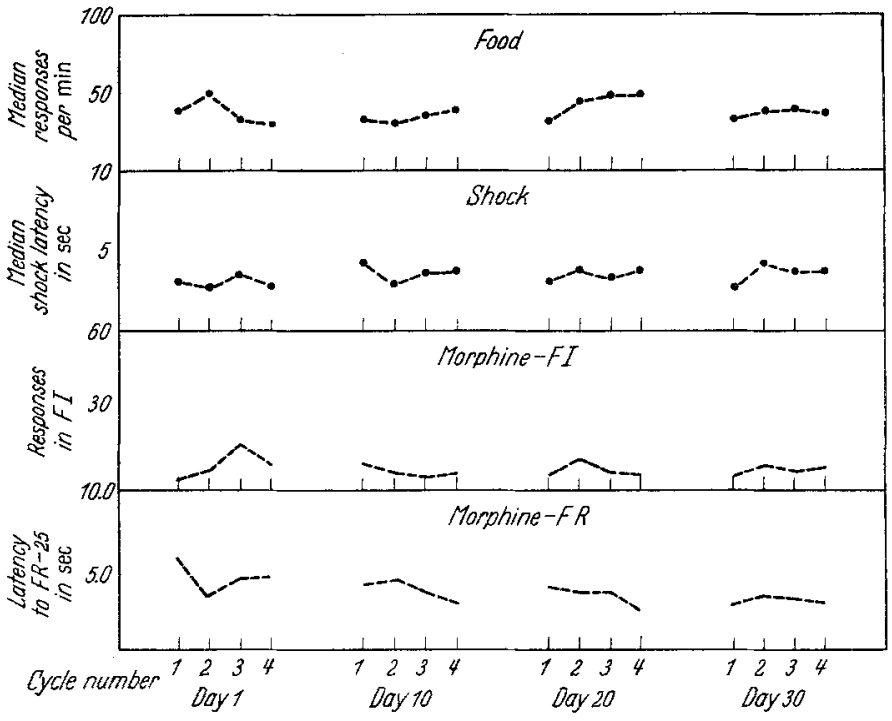

Fig. 3, Baseline performance on food-reinforced, shock avoidance, and fixed interval-fixed ratio drug-reinforced behaviors, over a period of 30 days
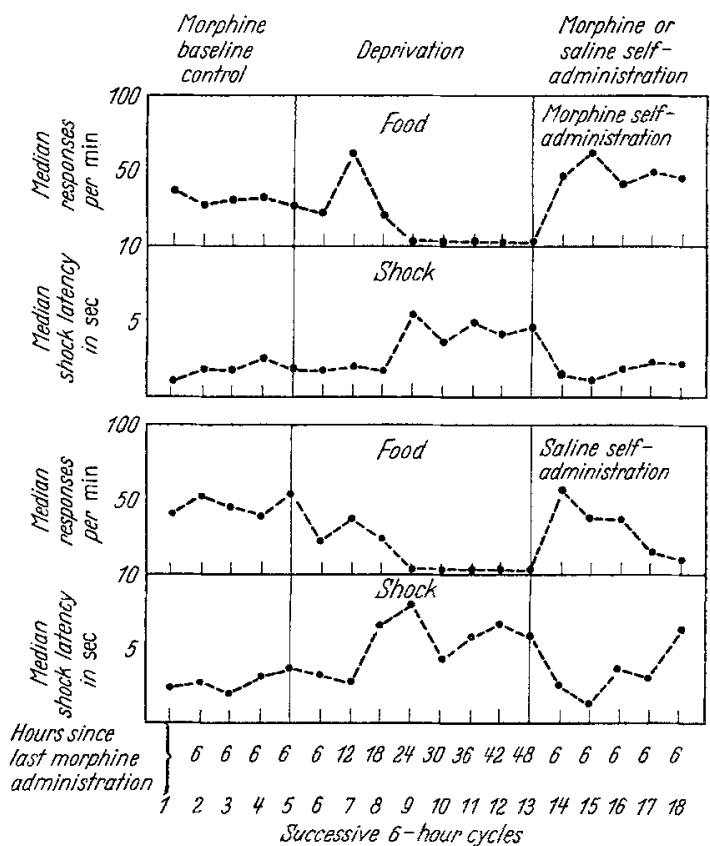

Fig. 4. Food and avoidance behaviors under conditions of morphine baseline control, 48 hours without the opportunity to self-administer morphine, followed by (upper graph) return to morphine baseline control procedure, or (lower graph) return to baseline condition with saline substituted for morphine self-administration 
with consistently the same result. A placebo effect was demonstrated for the infusion process by substitution of saline for morphine during the 60 second red light on the first self-administration opportunity after 48 hours abstinence. This effect is illustrated in the lower half of Fig. 4. The animals began working again immediately after the saline infusion. However, their food and shock performance showed progressive disruption as time without the drug increased.

'The subjects' FI-FR performance in these 4 periods in which saline was substituted for morphine showed no progressive changes indicative of extinction.

\section{Discussion}

The reinforcing effects of morphine to physically dependent animals has been well established. The present investigation has examined the properties of a more complex sequence of behaviors maintained by morphine administration, in relation to other aspects of the organism's behavior.

A two-member behavioral chain leading to the intravenous infusion of morphine was maintained with great reliability for 6 months. The FI-FR chain proved to be a reliable and sensitive index of the morphine deprivation conditions. The similarity of the effects of deprivation from morphine and pretreatment with the morphine antagonist, nalorphine, on the FI-FR chain, demonstrates the value of this procedure for the assessment of both environmental and pharmacological variables. Morphine pretreatment further illustrates the sensitivity of the chain to deprivation conditions. The functional relationship between the morphine pretreatment dosage and the characteristics of the subsequent FI-FR behavior provides a direct statement of the relationship between the degree of motivation and the animal's propensity to work for the drug.

Abstinence was found to produce changes not only in the morphine reinforced behavior, but also other behaviors essential to the animal's maintenance. Profound disruption of food and shock avoidance bebaviors was produced by 48 hours deprivation, with an immediate return to baseline conditions following a single morphine self-administration. The same immediate return to baseline behavioral levels was observed when saline was substituted for morphine in the first red light infusion period following 48 hours of deprivation. This placebo effect was transitory as indicated by the fact that succeeding self-administered saline infusions were seen to be associated with progressive disruption of the food and. avoidance behaviors. The fact that the saline infusion, in conjunction with the red light, maintained the FI-FR sequence for four periods suggests the conditioned reinforcing properties of stimuli previously associated with the morphine infusion reinforcement. Thus, the degree 
to which the physically dependent animal effectively deals with its environment is controlled, not only by the drug but also by the stimulus conditions associated with the prior self-administration of the drug.

\section{Summary}

1. A fixed interval-fixed ratio chain of behavior was maintained for periods as long as 6 months by intravenously administered morphine.

2. The morphine reinforced FI-FR chain was found to be a sensitive and reliable index of motivational changes induced by drug deprivation, nalorphine antagonism and pretreatment with morphine.

3. Profound behavioral disruption occurred in both shock avoidance and food reinforced ratio behavior under conditions of drug deprivation.

4. The behavioral disruption of the food reinforced and shock avoidance behaviors was ameliorated by a single self-administration of morphine. Substitution of saline for the morphine solution produced a transitory placebo effect characterized by an immediate return of the food and avoidance behaviors to their pre-deprivation baseline conditions, but followed by progressive disruption as time without the drug increased.

\section{References}

Davis, W. M., and J. R. Nichols: Physical dependence and sustained opiatedirected behavior in the rat. Psychopharmacologia (Berl.) 3, 139 (1962).

Nrсноц, J. R., and W. M. Davrs: Drug addiction II. Variation of addiction. J. Amer. pharm. Ass. 48, 259 (1959).

- C. P. Headlee and H. W. Coppock: Drug addiction 1. Addiction by escape training. J. Amer. pharm. Ass. 45, 788 (1956).

Nemarn, W. H., C. R. Schuster and T. Thompson: A surgical preparation for chronic intravenous infusion in Rhesus monkeys. University of Maryland, Laboratory of Psychopharmacology Technical Report No. 62-39, September, 1962.

Schuster, C. R., and T. Thompson: Self-administration of morphine in physically dependent monkeys. University of Maryland, Laboratory of Psychopharmacology Technical Report No. 62-29, July, 1962.

Serevers, M. H.: Opiate addiction in monkeys; methods of study. J. Pharmacol. exp. Ther. 56, 147 (1936).

- Ch. 19 in Pharmacology in Medicine (V. A. DriLl, ed.). New York: MoGrawHill Book Co. 1958.

Wwers, J. R.: Self-maintained morphine "addiction" - A method for chronic programmed intravenous injection in unrestrained rats. Fed. Proc. 20, 397 (1961) (abst).

- Experimental morphine addiction: Method for automatic intravenous injections in unrestrained rats. Science 138, 143 (1962).

Dr. Ch. R. Schuster,

Department of Pharmacology, University of Michigan, Ann Arbor, Michigan, USA 